\title{
EXPERIMENTO DIDÁTICO SOBRE CROMATOGRAFIA GASOSA: UMA ABORDAGEM ANALÍTICA E AMBIENTAL
}

\author{
José Carlos P. Penteado*, Dulce Magalhães e Jorge C. Masini \\ Instituto de Química, Universidade de São Paulo, CP 26077, 05513-970 São Paulo - SP, Brasil
}

Recebido em 16/10/07; aceito em 16/4/08; publicado na web em 3/10/08

\begin{abstract}
DIDACTIC EXPERIMENT ON GAS CHROMATOGRAPHY: AN ENVIRONMENTAL AND ANALYTICAL APPROACH. This paper describes an experiment to teach the principles of gas chromatography exploring the boiling points and polarities to explain the elution order of a series of alcohols, benzene and $n$-propanone, as well as to teach the response factor concept and the internal standard addition method. Retention times and response factors are used for qualitative identification and quantitative analysis of a hypothetical contamination source in a simulated water sample. The internal standard $n$-propanol is further used for quantification of benzene and $n$-butanol in the water sample. This experiment has been taught in the instrumental analysis course offered to chemistry and oceanography students.
\end{abstract}

Keywords: benzene; internal standard; contamination.

\section{INTRODUÇÃO}

Entre os métodos de separação, a cromatografia tem grande aplicabilidade em áreas tão diversas como ambiental, farmacêutica, análises clínicas, medicina legal e outras. A cromatografia permite separar e quantificar componentes com características físico-químicas muito semelhantes, tais como dioxinas e furanos, ${ }^{1}$ em misturas complexas. O limite de detecção obtido pela cromatografia pode ser cerca de 100 a 1000 vezes menor que aquele obtido por outros métodos de separação. ${ }^{2}$

O processo de separação ocorre através da distribuição dos componentes da amostra entre duas fases: uma fase fixa de grande área superficial (fase estacionária), que é percolada por um fluido (fase móvel) em uma direção definida. ${ }^{3}$ A fase móvel pode ser um gás inerte, um líquido ou um fluido supercrítico, dependendo do tipo de cromatografia utilizada. A fase estacionária deve ser imiscível com a fase móvel, podendo ser colocada numa coluna ou depositada em uma superfície plana. As fases móvel e estacionária são escolhidas de modo que os componentes da amostra se distribuam entre elas de modo distinto. Os analitos fortemente retidos pela fase estacionária movem-se mais lentamente na fase móvel e, conseqüentemente, são eluídos posteriormente aos componentes com baixa interação com a fase estacionária. Essa retenção seletiva dos componentes da amostra pela fase estacionária é uma importante característica e resulta em migrações diferenciadas dos compostos de interesse. De acordo com o estado físico da fase móvel utilizada, a cromatografia em coluna pode ser classificada em cromatografia gasosa (gás inerte), cromatografia líquida $^{4}$ (líquido) ou cromatografia com fluido supercrítico (fluido no estado supercrítico). ${ }^{3,5}$

Com mais de um século de existência, a cromatografia, iniciada por Michael S. Tswett, ${ }^{6}$ participou direta ou indiretamente de pesquisas que foram laureadas com 12 prêmios Nobel (1937 a 1972). ${ }^{3}$

\section{Cromatografia gasosa}

A cromatografia gasosa ${ }^{8,9}$ é uma das mais importantes técnicas analíticas disponíveis atualmente. Em curto espaço de tempo tornou- se a principal técnica para separação e determinação de compostos voláteis e/ou volatilizáveis. O poder de resolução excelente alcançado permite a determinação de dezenas de compostos diferentes em matrizes complexas. ${ }^{10}$ Outro diferencial da técnica vem a ser sua grande sensibilidade e elevada detectabilidade. Por exemplo, o limite de detecção para o pesticida halogenado Lindane pode atingir valores tão baixos quanto $10^{-16} \mathrm{~mol} \mathrm{~mL}^{-1}$ empregando-se o detector de captura de elétrons. ${ }^{11}$ A cromatografia gasosa pode ser aplicada em amostras gasosas, líquidas ou sólidas, desde que os analitos sejam voláteis ou possam ser volatilizados sem sofrer decomposição térmica.

A cromatografia gasosa classifica-se, de acordo com a natureza da fase estacionária, em cromatografia gás-sólido (CGS) e cromatografia gás-líquido (CGL). A fase estacionária pode ser um sólido (CGS) ou um líquido imobilizado sobre um suporte inerte (CGL), sendo que a CGL é a mais versátil e seletiva forma de cromatografia a gás, devido à grande diversidade de fases líquidas disponíveis.

Em linhas gerais, os principais componentes de um cromatógrafo a gás são: cilindro de gás, injetor, forno, coluna, detector, sistema de controle do instrumento e aquisição de dados.

Inicialmente a amostra é introduzida no injetor aquecido (com o auxílio de uma micro-seringa), onde é vaporizada e transferida com o auxílio do gás de arraste para a coluna cromatográfica colocada dentro de um forno pré-aquecido. Os componentes da amostras são eluídos e conduzidos para o detector conectado na saída da coluna. $\mathrm{O}$ detector emite um sinal elétrico que é registrado graficamente sob a forma de picos.

Trabalhos didáticos envolvendo cromatografia gasosa redigidos em língua portuguesa têm sido pouco explorados neste periódico. Com o intuito de colaborar com a divulgação e ensino da cromatografia, apresentamos uma proposta de experimento para a disciplina de análise instrumental com enfoque na introdução à cromatografia gasosa para alunos de graduação em Química e áreas correlatas. Este experimento explora a investigação da contaminação por solventes orgânicos em águas. Para isso, os alunos deverão investigar quatro fontes de contaminação em duas amostras de água. Entre os solventes adicionados destaca-se o benzeno, uma substância reconhecidamente carcinogênica, considerada a quinta substância de maior risco, segundo os critérios do programa das Nações Unidas. ${ }^{7}$ 


\section{PARTE EXPERIMENTAL}

\section{Instrumentação}

Foi utilizado o cromatógrafo a gás modelo Varian GC-3800 (Palo Alto, CA, USA) com detector de ionização em chama (250 $\left.{ }^{\circ} \mathrm{C}\right)$, injetor $\left(250{ }^{\circ} \mathrm{C}\right)$ com divisão de fluxo na razão $1: 25$, coluna capilar HP-Innowax (30 m x 0,25 mm x 0,25 $\mu \mathrm{m})(\mathrm{J} \& \mathrm{~W}$, USA) empregando $\mathrm{N}_{2}$ como gás de arraste, com fluxo constante de $1 \mathrm{~mL}$ $\mathrm{min}^{-1}$. A temperatura inicial foi de $80^{\circ} \mathrm{C}(1 \mathrm{~min})$, seguida por taxa de aquecimento de $10^{\circ} \mathrm{C} \mathrm{min}-1$ até $120^{\circ} \mathrm{C}$, com tempo total de corrida de 6 min. $\mathrm{O}$ volume de injeção foi igual a 1,0 $\mu \mathrm{L}$. A aquisição e o processamento de dados foram feitos com o programa Star Advanced versão 4.52 da Varian.

\section{Reagentes e soluções}

Todos os reagentes utilizados (isobutanol, $n$-propanol, $n$-propanona, benzeno, $n$-pentanol) foram procedentes da Merck Brasil (Rio de Janeiro, RJ, Brasil) com teores maiores que 98\%. A água desionizada foi obtida pelo sistema Milli-Q, apresentando resistividade maior que $18 \mathrm{M} \Omega \mathrm{cm}^{-1}$. Foram preparadas as seguintes misturas de soluções de referência, conforme mostrado na Tabela 1: A - isobutanol (500 $\mathrm{mg} \mathrm{L}^{-1}$ ) e $n$-pentanol (500 mg L-1); B $-n$-propanona $\left(500 \mathrm{mg} \mathrm{L}^{-1}\right) \mathrm{e}$ $n$-pentanol (500 $\left.\mathrm{mg} \mathrm{L}^{-1}\right)$; C - benzeno (350 $\left.\mathrm{mg} \mathrm{L}^{-1}\right)$ e $n$-butanol (81 mg L $\left.{ }^{-1}\right)$; D - benzeno (88 $\mathrm{mg} \mathrm{L}^{-1}$ ) e $n$-butanol (324 mg L $\mathrm{m}^{-1}$ ); padrão interno (PI) $n$-propanol (500 $\left.\mathrm{mg} \mathrm{L}^{-1}\right)$ e as amostras $\mathrm{W}$ - benzeno (175 mg L $\left.\mathrm{m}^{-1}\right)$ e $n$-butanol (40 $\left.\mathrm{mg} \mathrm{L}^{-1}\right)$ e $\mathrm{H}$ - benzeno $\left(44 \mathrm{mg} \mathrm{L}^{-1}\right)$ e n-butanol (162 $\left.\mathrm{mg} \mathrm{L}^{-1}\right)$.

Tabela 1. Tempos de retenção $\left(\mathrm{t}_{\mathrm{r}}\right)$, pontos de ebulição ${ }^{13}(\mathrm{PE})$ e constante dielétrica $(\varepsilon)$ dos analitos presentes nas soluções A, B, C, D, W e H

\begin{tabular}{llrrrrrrrr}
\hline Analitos & $\mathrm{t}_{\mathrm{r}}(\min )$ & $\mathrm{PE}\left({ }^{\circ} \mathrm{C}\right)$ & $\varepsilon$ & $\mathrm{A}$ & $\mathrm{B}$ & $\mathrm{C}$ & $\mathrm{D}$ & $\mathrm{W}$ & $\mathrm{H}$ \\
\hline n-propanona & 2,319 & 56,05 & 21,01 & & $\mathrm{x}$ & & & & \\
benzeno & 2,734 & 80,09 & 2,28 & & & $\mathrm{x}$ & $\mathrm{x}$ & $\mathrm{x}$ & $\mathrm{x}$ \\
$n$-propanol & 3,147 & 97,20 & 20,80 & & & & & & \\
isobutanol & 3,562 & 107,89 & 17,93 & $\mathrm{x}$ & & & & & \\
$n$-butanol & 4,089 & 117,73 & 17,84 & & & & & & \\
$n$ - pentanol & 5,449 & 137,98 & 15,13 & $\mathrm{x}$ & $\mathrm{x}$ & & & & \\
\hline
\end{tabular}

\section{Procedimentos}

Este experimento se compõe das etapas qualitativa e quantitativa, sendo que a primeira se baseia na identificação dos compostos utilizando o tempo de retenção, enquanto a segunda etapa consiste na determinação da concentração dos analitos a partir das áreas de pico.

\section{Etapa qualitativa}

Foram injetadas, seqüencialmente, 1,0 $\mu \mathrm{L}$ das soluções A, B, C, $\mathrm{D}, \mathrm{W}$ e $\mathrm{H}$ diluídas na razão de 1:10 em água desionizada. A seguir foram atribuídos os picos observados nos cromatogramas de cada solução. A identificação de cada componente foi realizada pelos seus respectivos tempos de retenção $\left(\mathrm{t}_{\mathrm{r}}\right)$, que representam o tempo gasto desde o momento da injeção até a saída do ponto máximo do pico.

\section{Etapa quantitativa}

Com auxílio de uma pipeta volumétrica transferiu-se $1,00 \mathrm{~mL}$ da solução C e 1,00 mL da solução de PI (500 $\left.\mathrm{mg} \mathrm{L}^{-1}\right)$ para um balão volumétrico de $10 \mathrm{~mL}$, completando o volume com água desionizada. O mesmo procedimento foi repetido para as soluções D, W e
H. Um volume de 1,0 $\mu \mathrm{L}$ de cada uma destas soluções foi injetado seqüencialmente no cromatógrafo a gás para a determinação das áreas de pico correspondentes a cada um dos analitos, previamente identificados pelos respectivos tempos de retenção.

\section{RESULTADOS E DISCUSSÃO}

\section{Etapa qualitativa}

Os resultados de tempo de retenção médios obtidos na etapa qualitativa são apresentados na Tabela 1 em comparação com a composição das soluções, bem como com os pontos de ebulição e constantes dielétricas dos componentes das misturas. Observa-se que a cromatografia gasosa é uma técnica com grande aplicação na determinação simultânea de compostos voláteis e semi-voláteis, tais como álcoois, ${ }^{12}$ cetonas e hidrocarbonetos. A separação cromatográfica das diferentes famílias de compostos orgânicos foi possível devido às características físico-químicas dos analitos, tais como ponto de ebulição e constante dielétrica, ${ }^{13}$ parâmetro este que é relacionado com a polaridade das substâncias e que determina a afinidade pela fase estacionária (polar, no presente experimento). Entre todos os componentes, a $n$-propanona é o primeiro componente a ser eluído apesar de apresentar a constante dielétrica mais elevada. Este fato ocorre devido principalmente a sua alta volatilidade $\left(56,05^{\circ} \mathrm{C}\right)$. $\mathrm{O}$ segundo componente é o benzeno, que, entre os compostos estudados, é o mais apolar ( $\varepsilon 2,28)$. Essa característica faz com que tenha baixa afinidade pela fase estacionária (polar), sendo eluído rapidamente. A partir do benzeno, temos o grupo dos alcoóis com 3 a 5 átomos de carbono na cadeia principal. Esses alcoóis têm características polares ( $\varepsilon 15,13-20,8)$, sofrendo uma maior interação com a fase estacionária. Entretanto, para estes alcoóis, o ponto de ebulição vem ser a característica dominante na ordem de eluição.

Na solução A (isobutanol e $n$-pentanol), o primeiro componente a ser eluído é o isobutanol. O isobutanol apresenta maior constante dielétrica que o $n$-pentanol, de modo que, baseando-se apenas nos dados de polaridade, se esperaria que o isobutanol fosse o último composto a ser eluído da coluna. Entretanto, o ponto de ebulição do isobutanol é cerca de $30^{\circ} \mathrm{C}$ menor que o $n$-pentanol, de modo que para compostos cuja diferença de polaridade não seja elevada, a volatilidade se torna o principal parâmetro que define a ordem de eluição. No caso da solução $\mathrm{B}$ (n-propanona e $n$-pentanol), a $n$-propanona é o primeiro componente a ser eluído, seguido pelo $n$-pentanol. Com base apenas na polaridade, a $n$-propanona deveria ser eluída por último, pois apresenta uma constante dielétrica maior que o $n$-pentanol e, portanto, possui uma maior afinidade com a fase estacionária. No entanto, é eluída primeiro, pois seu ponto de ebulição é cerca de três vezes menor que o do $n$-pentanol. Em relação às soluções $\mathrm{C}, \mathrm{D}, \mathrm{W}$ e $\mathrm{H}$ (benzeno e $n$-butanol), o benzeno é eluído antes do $n$-butanol. Nesse caso os valores de constante dielétrica e ponto de ebulição justificam a ordem de eluição, pois o benzeno é menos polar e tem ponto de ebulição menor que o $n$-butanol.

O princípio do funcionamento do detector de ionização de chama (DIC) baseia-se na combustão dos compostos na chama. Os compostos não suscetíveis à combustão, como água, $\mathrm{CO}_{2}$ e compostos inorgânicos em geral, não apresentam sinal mensurável neste detetor. ${ }^{3} \mathrm{O}$ sinal elétrico enviado pelo detector é registrado graficamente sob a forma de pico, cuja área é proporcional à concentração do analito. $\mathrm{O}$ perfil cromatográfico da solução C e da amostra W, bem como da solução D e amostra H são semelhantes (Figura 1). Na Figura 1a, o pico com maior intensidade vem a ser o benzeno (solução $\mathrm{C}$ e amostra $\mathrm{W}$ ), enquanto que na Figura $1 \mathrm{~b}$ isso ocorre com o $n$-butanol (solução D e amostra H). Essa observação pode ser constatada numericamente na Tabela 2 , onde estão descritos os resultados das áreas obtidas nos cromatogramas e as relações de áreas entre benzeno e $n$-butanol para as soluções C e D e as amostras W e H. 

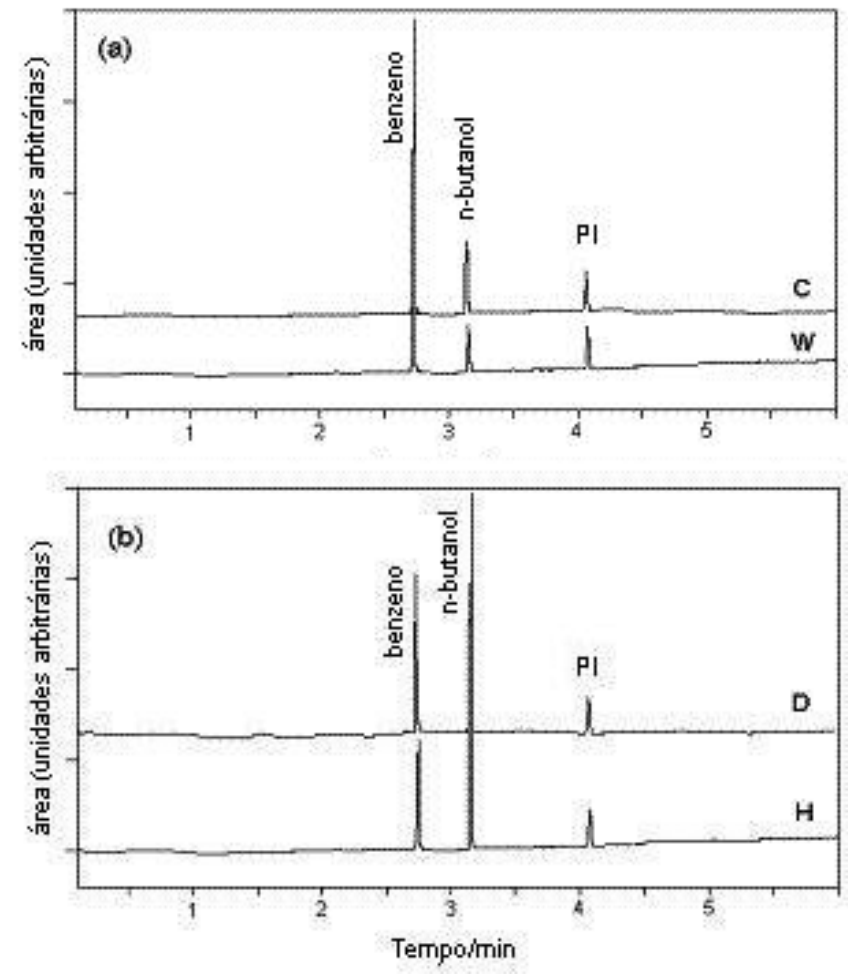

Figura 1. Cromatogramas obtidos da amostra $W$ em comparação com a solução de referência $C(\mathrm{la})$ e amostra da amostra $\mathrm{H}$ em comparação com a solução de referência $D(1 b)$

Uma vez que a relação de área entre o benzeno e o $n$-butanol da solução C é 7,49, pode-se inferir que é esta a fonte de contaminação da amostra W, para a qual a relação de área destes dois compostos é de 7,53. De modo similar, pode se inferir que a solução D (relação 0,47) é a fonte de contaminação da amostra $\mathrm{H}$ (relação 0,40$)$.

\section{Etapa quantitativa}

A quantificação dos analitos foi feita pelo método de adição de padrão interno, que consiste em adicionar uma quantidade conhecida de uma substância (padrão interno) na amostra a ser analisada e no padrão, relacionando então as duas áreas obtidas. ${ }^{14} \mathrm{O}$ padrão interno aumenta a precisão dos resultados ao minimizar as incertezas introduzidas especialmente pela injeção da amostra, mas também por variações de vazão e das condições da coluna. ${ }^{15-17}$ Neste experimento o padrão interno selecionado foi o $n$-propanol cujo tempo de retenção está depois do benzeno e $n$-butanol.

A Tabela 2 mostra os resultados obtidos dos fatores de resposta dos analitos benzeno e $n$-butanol para os padrões $\mathrm{C}$ e $\mathrm{D}$ e as concentrações calculadas para as amostras W e H.

\section{Concentrações nas amostras $W$ e $H$}

A determinação das concentrações de benzeno e $n$-butanol foi feita a partir da adição de padrão interno, no caso $n$-propanol, na concentração de $500 \mathrm{mg} \mathrm{L}^{-1}$. Para isso, a primeira etapa envolve a obtenção do chamado fator de resposta (F) do detector para os dois analitos, o qual é definido como: ${ }^{18}$

$F=\left(\frac{\frac{A_{P}}{C_{P}}}{l}\right) /\left(\frac{A_{P I}}{C_{P I}}\right)$

onde $A_{P}$ e $C_{P}$ são a área de pico e concentração de um padrão do analito (benzeno ou $n$-butanol), obtidos com os cromatogramas das soluções $\mathrm{C}$ ou $\mathrm{D}$, enquanto $A_{P I}$ e $C_{P I}$ são a área de pico e concentração do padrão interno ( $n$-propanol $500 \mathrm{mg} \mathrm{L}^{-1}$ ), respectivamente. De posse do fator de resposta, e reescrevendo a Equação 1, a concentração do analito na amostra pode ser calculada a partir da Equação 2:

$C_{X}=\frac{A_{X} \cdot C_{P I}}{F_{X} \cdot A_{P I}}$

onde $C_{x}$ é a concentração do analito $\mathrm{X}$ na amostra desconhecida, $\mathrm{F}_{\mathrm{X}}$ é o fator de resposta para este analito $\mathrm{e} \mathrm{A}_{\mathrm{X}}$ é a sua respectiva área de pico, obtida dos cromatogramas das soluções $\mathrm{W}$ ou $\mathrm{H}$.

Embora teoricamente os fatores de respostas obtidos nas soluções padrões $\mathrm{C}$ e $\mathrm{D}$ devessem ser os mesmos, na prática houve uma diferença para o benzeno e $n$-butanol (Tabela 2). Essa variação ocorreu provavelmente devido a diferenças entre as concentrações dos analitos nas soluções C e D. Assim, o cálculo das concentrações dos analitos nas soluções das amostras $\mathrm{W}$ e $\mathrm{H}$ foi feito empregando os fatores de resposta das soluções de referência $\mathrm{C}$ e $\mathrm{D}$, respectivamente.

As concentrações obtidas de benzeno e $n$-butanol nas amostras $\mathrm{W}$ e $\mathrm{H}$ foram próximas aos dos valores esperados, conforme mostrado na Tabela 2, indicando que o método de padronização interna foi eficiente na quantificação dos analitos. As concentrações de benzeno encontradas neste experimento são elevadas quando comparadas com alguns valores reportados na literatura. ${ }^{20}$ Porém, recentemente foram divulgados acidentes desastrosos no Rio Songhua (China), onde ocorreram vazamentos de enormes quantidades de benzeno e nitrobenzeno. ${ }^{21}$ Este acidente afetou o abastecimento de milhões de habitantes tendo um custo estimado de remediação de US\$ 3,8 bilhões. ${ }^{22}$

\section{CONCLUSÕES}

O experimento proposto pode ser facilmente implementado em laboratórios de ensino de graduação em Química e áreas correlatas. Os pontos discutidos fornecem ao docente flexibilidade na abordagem didática e despertam o interesse dos alunos na aplicabilidade

Tabela 2. Resultados de áreas e suas relações, bem como as concentrações obtidas dos analitos benzeno e $n$-butanol

\begin{tabular}{|c|c|c|c|c|c|c|c|c|}
\hline \multirow[t]{2}{*}{ Solução } & \multicolumn{3}{|c|}{ Áreas } & \multirow{2}{*}{$\begin{array}{c}\text { Relação } \\
\text { benzeno/n-butanol }\end{array}$} & \multicolumn{2}{|c|}{ Fator de Resposta } & \multicolumn{2}{|c|}{$\begin{array}{c}\text { Concentração das amostras } \\
\qquad\left(\mathrm{mg} \mathrm{L}^{-1}\right)\end{array}$} \\
\hline & benzeno & $n$-butanol & PI & & benzeno & $n$-butanol & benzeno & $n$-butanol \\
\hline $\mathrm{C}$ & 3328 & 444 & 241 & 7,49 & 19,7273 & 11,3724 & & \\
\hline W & 1429 & 190 & 210 & 7,53 & & & $172,5 \pm 0,2$ & $39,7 \pm 0,1$ \\
\hline D & 649 & 1377 & 196 & 0,47 & 18,8138 & 10,8418 & & \\
\hline $\mathrm{H}$ & 304 & 741 & 191 & 0,40 & & & $42,3 \pm 0,3$ & $178,9 \pm 0,5$ \\
\hline
\end{tabular}

${ }^{a}$ As concentrações esperadas de benzeno e $n$-butanol eram, respectivamente, 175 e $39,7 \mathrm{mg} \mathrm{L}^{-1}$ na amostra W e 44 e $162 \mathrm{mg} \mathrm{L}{ }^{-1}$ na amostra $\mathrm{H}$. 
da cromatografia gasosa. Neste experimento foi feita uma simulação de contaminação de benzeno em água, pois em condições reais a concentração máxima de benzeno em água potável permitida pela legislação é de $5 \mu \mathrm{g} \mathrm{L}^{-1}$ (CETESB). ${ }^{19}$

\section{AGRADECIMENTOS}

Os autores agradecem à FAPESP pelo apoio financeiro.

\section{REFERÊNCIAS}

1. Pavuk, M.; Patterson, D. G.; Turner, W. E.; Deedham, L. L.; Ketchum, N. S.; Chemosphere 2007, 68, 62.

2. Harvey, D.; Modern Analytical Chemistry, McGraw-Hill: New York, 2000.

3. Skoog, D. A.; Holler, F. J.; Nieman, T. A.; Princípios de Análise Instrumental, $5^{\mathrm{a}}$ ed., Bookman: Porto Alegre, 2002.

4. Manfredi, A. L. P.; Collins, C. H.; Manfredi, J. E.; Quim. Nova 1983, 6, 103.

5. Lanças, F. M.; Cromatografia em Fase Gasosa, Acta: São Carlos, 1993.

6. Ettre, L. S.; Chromatographia 2000, 51, 7.

7. McMichael, A. J.; Carcinogenicity of benzene, toluene and xylene: epidemiological and experimental evidence, IARC Sci Publ., 1988.
8. Collins, R. L.; Aquino Neto, F. R.; Silva, J. R. P.; Quim. Nova 1988, 11, 4.

9. Collins, C. H.; Berg, R. G.; Valente, A. L. P.; Kugler, W.; Murta, A. L. M.; Quim. Nova 1982, 5, 115.

10. Focant, J. F.; Sjoin, A. ; Patterson, D. G. J.; J. Chromatogr., A 2004, 1040, 227

11. Grob, R. L.; Modern Practice of Gas Chromatography, $4^{\text {th }}$ ed., John Wiley \& Sons: Hooboken, 2004.

12. Musshoff, F.; J. Chromatogr., B 2002, 781, 457.

13. Handbook Chemistry and Physics, $87^{\text {th }}$ ed., CRC Press: San Francisco, 2006.

14. Ciola, R.; Fundamentos da Cromatografia a Gás, Edgard Blücher: São Paulo, 1985

15. Ribani, M.; Bottoli, C. B. G.; Collins, C. H.; Jardim, I. C. S. F.; Melo, L. F. C.; Quim. Nova 2004, 27, 771.

16. Magge, J. A.; Antony, H. C.; J. Chem. Educ. 1999, 76, 252.

17. Cazes, J.; Encyclopedia of Chromatography, Marcel Dekker: New York, 2004.

18. Rice, G. W.; J. Chem. Educ. 1987, 64, 1055.

19. Ministério da Saúde, Portaria no 518, 25/3/2004, cap. 4, art. 14; Norma de qualidade de água para consumo humano.

20. Owens, J. E.; Niermeyer, E. D.; Environ. Pollut. 2006, 140, 506.

21. Zhu, L.; Ma, B.; Zhang, L.; Zhang, L.; Chemosphere 2007, 68, 10.

22. http://english.gov.cn/2006-01/10/content_152926.htm, acessada em Outubro 2007. 